\title{
Opportunities for Time-resolved Dynamic CT Imaging in the Laboratory
}

\author{
Arno Merkle ${ }^{1}$, Marijn Boone ${ }^{1}$, Denis Van Loo ${ }^{1}$, Jan Dewanckele ${ }^{1}$ and Frederik Coppens ${ }^{1}$ \\ 1. TESCAN XRE, Ghent, Belgium.
}

Time-resolved 3D imaging with X-rays has rapidly emerged as an essential technique to understand materials evolution, facilitating in situ investigations ranging from mechanical deformation to fluid flow in porous materials and beyond. Imaging of dynamic processes is one of the key applications at synchrotron facilities, pushing the time resolution more and more down with quite some success. However, access to those facilities is often limited and operational cost are quite high.

In the laboratory, image quality and spatial resolution have been significantly improved in recent years, however often at a cost of temporal resolution. Recent developments and experimental design at TESCAN XRE have made it possible to visualize and inspect dynamic process in the laboratory with a tomographic temporal resolution below 10 seconds. In this study we explore the challenges and innovations that have led to this capability.

Dynamic acquisitions however, generate vast amounts of raw projection data, which need to be reconstructed, further post processed and eventually quantified. It is therefore essential to devise workflow strategies to quickly identify the interesting moments prior to reconstruction to optimize the amount of data that is generated, but also incorporate the added time dimension in the 3D analysis workflow to improve image quality. Challenges and possibilities in dynamic micro CT imaging in the laboratory will be demonstrated here across materials science, life science and geoscience applications.

A gantry-based micro-CT system (TESCAN DynaTOM) is employed to demonstrate the range of applications possible by continuous dynamic scanning in a laboratory setting. This architecture, consisting of a source and detector rotating in a horizontal plane around a stationary specimen affords maximum flexibility for in situ experiments, especially those of delicate structures, often involving continuously moving elements or fluid flow.

Results consist of several examples from materials science, geology and biological samples. In Figure 1, morphological evolution of a delicate foam structure is investigated during collapse. After 4D acquisition, reconstruction and quantification, it was determined that the distribution of pore sizes differed substantially as a function of foam composition. Further results (Figure 2) represent another class of dynamic tomography results, consisting of fluid flow experiments performed in a custom-made X-ray transparent flow cell on artificial and geological porous structures. These multiphase flow experiments were focused on drainage processes, injection of non-wetting liquid (oil) in the pore network, and imbibition processes, injection of wetting phase (water) in the pore network. The experimental setup for both flow experiments was very similar and details can be found in Bultreys et al. [1]. The capabilities of dynamic reconstruction and the incorporation of temporal information in the 3D analysis of the pore space facilitate the automatic identification pore filling events and pinpoint these events in time. By reconstructing images from different time intervals and merging the appropriate temporal and local information for analysis, the speed and size of these events can be monitored. 
References:

[1] T Bultreys et al., Water Resources Research 51 (2015), p. 8668.

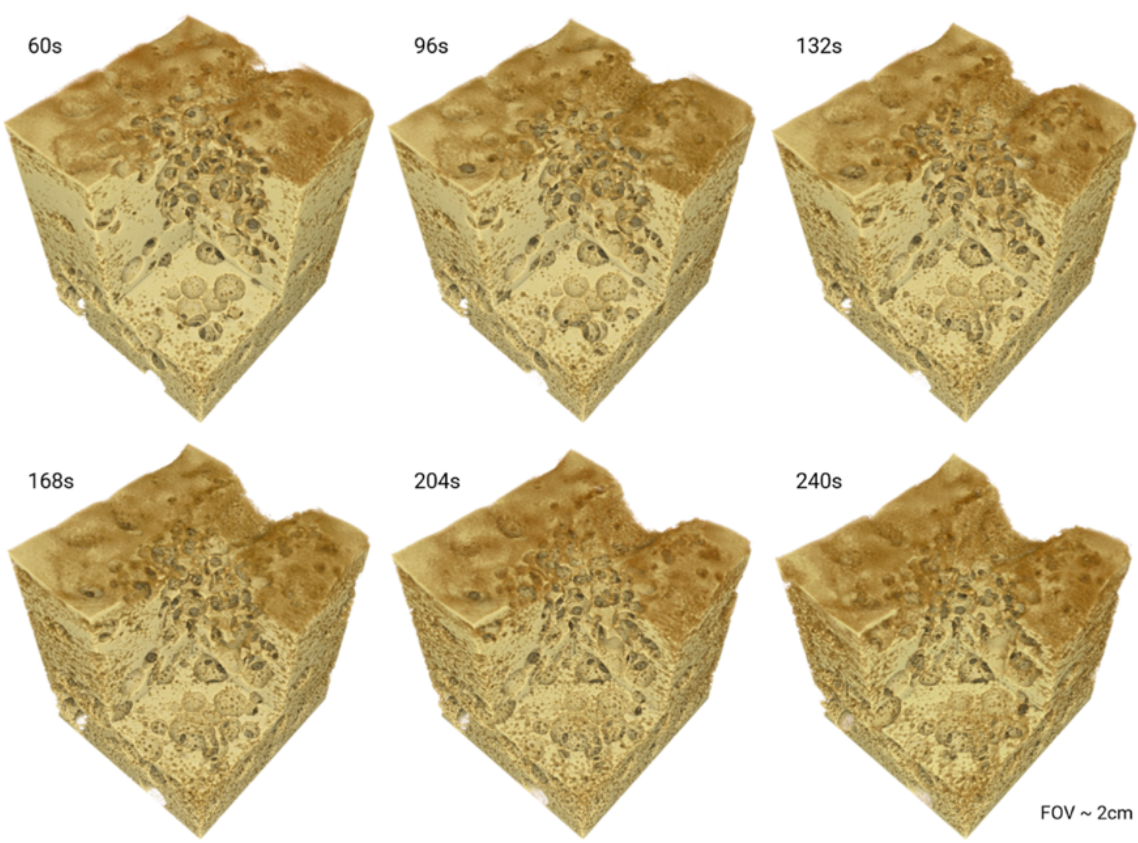

Figure 1. Foam collapse sequence of continuously acquired dynamic tomography dataset in the laboratory. Acquired on TESCAN DynaTOM with 9 second temporal resolution (per 360-degree rotation).

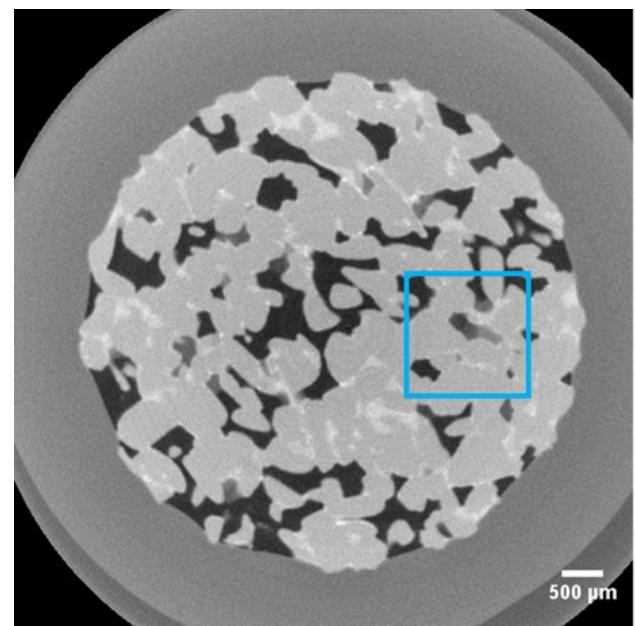

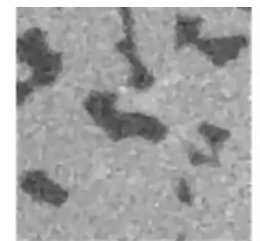

$\mathrm{t}=910 \mathrm{~s}$

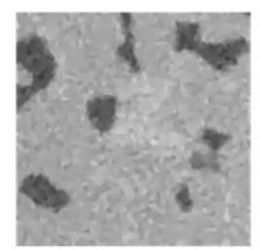

$\mathrm{t}=1680 \mathrm{~s}$

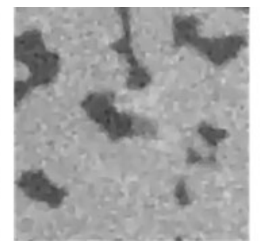

$\mathrm{t}=980 \mathrm{~s}$

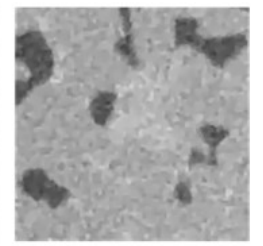

$\mathrm{t}=1750 \mathrm{~s}$

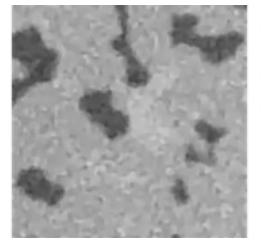

$\mathrm{t}=1050 \mathrm{~s}$

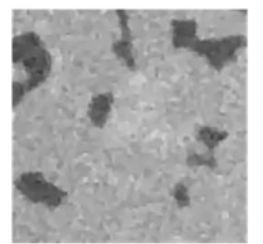

$\mathrm{t}=1820 \mathrm{~s}$

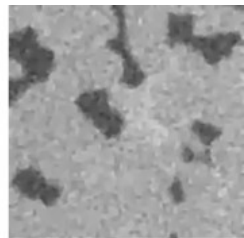

$\mathrm{t}=1120 \mathrm{~s}$

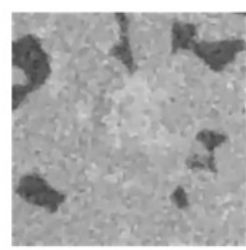

$\mathrm{t}=1890 \mathrm{~s}$

Figure 2. 2D virtual slice of $3 \mathrm{D}$ micro-CT data from a cylindrical volume (voxel size $13 \mu \mathrm{m}$ ) of a porous geological sample. Right: Detail of pore filling evolution during imbibition. Each reconstructed volume has a temporal resolution of 70 seconds. 